\title{
Enhancement of Memories by Systemic Administration of Insulin-Like Growth Factor II
}

\author{
Sarah A Stern ${ }^{1,2}$, Amy S Kohtz' ${ }^{1,3}$, Gabriella Pollonini' and Cristina M Alberini*,' \\ 'Center for Neural Science, New York University, New York, NY, USA; ${ }^{2}$ Graduate School of Biomedical Sciences, Icahn School of Medicine at \\ Mount Sinai, New York, NY, USA; ${ }^{3}$ Graduate Program in Psychology, University at Albany-SUNY, Albany, NY, USA
}

\begin{abstract}
To treat cognitive disorders in humans, new effective therapies that can be easily delivered systemically are needed. Previous studies showed that a bilateral injection of insulin-like growth factor II (IGF-II) into the dorsal hippocampus of rats or mice enhances fear memories and facilitates fear extinction. Here, we report that, in mice, systemic treatments with IGF-II given before training significantly enhance the retention and persistence of several types of working, short-term and long-term memories, including fear conditioning, object recognition, object placement, social recognition, and spatial reference memory. IGF-II-mediated memory enhancement does not alter memory flexibility or the ability for new learning and also occurs when IGF-II treatment is given in concert with memory retrieval. Thus IGF-II may represent a potentially important and effective treatment for enhancing human cognitive and executive functions. Neuropsychopharmacology (2014) 39, 2179-2190; doi:I0.1038/npp.2014.69; published online 23 April 2014
\end{abstract}

\section{INTRODUCTION}

Memory and cognitive impairments are associated with numerous diseases or deficits, including Alzheimer's disease, aging, dementias, anxiety/stress-related disorders, and autism spectrum disorder (ASD), and the need for novel therapies that may lead to memory/cognitive enhancement is extremely high. Furthermore, for rapid translation into clinical therapies, it is critical to identify effective and successful treatments that can be easily administered through systemic delivery. IGF-II is a polypeptide belonging to the insulin system that has an important role in normal somatic growth and development, tissue repair, and regeneration (Roth, 1988; Russo et al, 2005; Werther et al, 1998). An increase in IGF-II expression in the hippocampus is required for rat inhibitory avoidance (IA) memory formation (Chen et al, 2011) and mouse extinction learning (AgisBalboa et al, 2011), and a bilateral hippocampal injection of recombinant IGF-II enhances fear memory retention, prevents its forgetting (Chen et al, 2011) and facilitates extinction (Agis-Balboa et al, 2011). Although these findings are important as proofs of principle, to progress in the direction of establishing novel effective clinical treatments it is essential that the effect of IGF-II is tested with systemic routes of administration and on different types of memories, particularly those that are lost or impaired in aging, cognitive disorders, and neurodegeneration.

*Correspondence: Professor CM Alberini, Center for Neural Science, New York University, 4 Washington Place, New York, NY I0003, USA, Tel: + I 212998772 I, Fax: + I 212995401 I,

E-mail: ca60@nyu.edu

Received 24 October 2013; revised 19 February 2014; accepted 16 March 2014; accepted article preview online 19 March 2014
IGFs, including IGF-II, have been shown to cross the blood-brain barrier (BBB) in several species (Duffy et al, 1988; Reinhardt and Bondy, 1994), and IGF-II receptors are located on $\mathrm{BBB}$ capillaries, indicating a receptor-mediated transport system for IGF-II transcytosis across the BBB (Rosenfeld et al, 1987). Here, we used C57BL/6J mice to test the effect of an acute systemic injection of IGF-II on several types of short- and long-term memories, working memories, as well as on memory flexibility. We utilized both fear-based/passive tasks that measure inactive behaviors (eg, freezing), as well as non-aversive/active tasks that measure approach behaviors (eg, object investigation), to determine whether the effects of IGF-II are applicable to multiple types of temporal lobe-dependent memory. We also investigated the effect of systemically delivered IGF-II on extinction, working memory, and memory flexibility. Finally, we established the safety of the treatment and the effects on metabolic parameters.

\section{MATERIALS AND METHODS}

\section{Animals}

Adult male C57BL/6J mice (6-10 weeks of age, Jackson Laboratory, Maine, USA) were group-housed (except for mice that underwent hippocampal implants, who were singly housed following surgery) on a 12-hour (h) light/dark cycle, with ad libitum access to food (except for the Y-Maze experiment) and water. Experiments were performed during the light cycle. All protocols complied with the NIH Guide for the Care and Use of Laboratory Animals and were approved by the New York University Animal Welfare Committee. 


\section{Drugs}

Recombinant mouse IGF-II (R\&D, Minnesota, USA cat no. 792-MG) was dissolved in $0.1 \%$ bovine serum albuminphosphate-buffered saline (0.1\% BSA-PBS, pH 7.4) and administered subcutaneously (s.c.) in $0.3 \mathrm{ml}$. Anti-IGF-II receptor antibody (anti-IGF-IIR) or IgG control (both from R\&D) were dissolved in $1 \times$ PBS and injected bilaterally into the hippocampus at $20 \mathrm{ng} / \mu \mathrm{l}$. This concentration blocks $90 \%$ of binding to the IGF-II receptor in an in vitro binding assay (R\&D).

\section{Contextual/Auditory Fear Conditioning (CFC and AFC) and Extinction}

Mice were handled for 2-3 min per day for 5 days before training. The conditioning chamber consisted of a rectangular Perspex box $\left(30.5 \times 24.1 \times 21.0 \mathrm{~cm}^{3}\right)$ with a metal grid floor (Model ENV-008 Med Associates, St Albans, Vermont, USA) through which foot shocks were delivered via a constant current scrambler circuit. Freezing, defined as lack of movement besides heartbeat and respiration, was recorded every tenth second by trained observers blind to the experimental conditions. The percent of freezing across the total number of observations was calculated (Schrick et al, 2007).

CFC was carried out as described (Guedea et al, 2011). An unsignalled 2-second (s) $0.7 \mathrm{~mA}$ footshock was delivered after $2 \mathrm{~min}$ in the chamber, after which the mouse remained for one more min. During testing, the mouse was placed back into the conditioning chamber for $3 \mathrm{~min}$ in the absence of a footshock. AFC procedure was modified from Han et al (Han et al, 2009). After $2 \mathrm{~min}$ in the chamber, mice received one tone-shock pairing (30-s tone coterminating with a $0.7-\mathrm{mA}$ footshock) and, 1 min later, were returned to the homecage. Memory was tested in an alternate context. During testing, after $1 \mathrm{~min}$ in the new context (pre-tone) the tone was played for $2 \mathrm{~min}$. An observer blind to experimental procedures scored all of the experiments, and memory was measured as the percentage of time spent freezing during the 2-min tone or during the pre-tone. CFC extinction was performed as described (Guedea et al, 2011). Briefly, one day following CFC training, mice were placed into the conditioning chamber for 3 min each day for 5 or 9 days.

\section{Spontaneous Alternation}

Spontaneous alternation was carried out as previously described (Mandillo et al, 2008). The Y-Maze consisted of three black polycarbonate arms $\left(7.62 \times 12.7 \times 38.1 \mathrm{~cm}^{3}\right)$ with wells at the end of two arms for food rewards $(1.93 \mathrm{~cm}$ diameter $\times 1.27 \mathrm{~cm}^{2}$ deep). Mice were allowed to freely explore from the center of the maze for $10 \mathrm{~min}$. Spontaneous alternation was defined as successive entries into each of the three arms on overlapping triplet sets (eg, ABC, BCA, CAB, etc.). Percentage alternation was defined as the ratio of actual alternations (total alternations) to possible alternations (total arm entries -2$) \times 100$.

\section{Spatial Reference Memory in the Y-Maze}

Spatial reference memory in the The Y-Maze was carried out as previously described (Van der Borght et al, 2007). Mice were single-housed and food restricted for 1 week before and during testing by giving $1 / 2$ food pellet (Purina
Lab Diet 5001, Missouri, USA) plus 1 fruit loop (Kellogg's) each day. Habituation was identical to spontaneous alternation. During acquisition training, one arm (counterbalanced between animals) was designated as the 'correct' arm and baited with a fruit loop, which the mouse could eat. Mice were enclosed in the start arm for $1 \mathrm{~min}$ and then permitted to choose between the two arms. Acquisition training consisted of two blocks of 5 trials (10 trials total) per day for 2 days. Learning and memory were calculated as the percentage of correct arms chosen over each block of trials. Reversal training was identical to the initial training, with the 'correct' arm switched. An observer blind to experimental procedures scored all of the experiments.

\section{Novel-Object Recognition (nOR) and Object Placement (OP)}

nOR and OP were adapted from Dix and Aggleton (1999). Mice were handled for 2-3 min each day for 5 days before training. Objects were counterbalanced during training and testing. Mice were trained in a clean square homecage, free of bedding, containing two identical objects (Mega Bloks 120), and were permitted to interact freely for $3 \mathrm{~min}$ (nOR) or $5 \mathrm{~min}$ (OP). Four and $24 \mathrm{~h}$ later, mice were placed back into the homecage, and one object was replaced with a novel object (in nOR), or one object was moved to an alternate location (in $\mathrm{OP})$. In both cases, one object remained constant throughout all of training and testing, while the other was replaced. Memory was measured as the percentage of time spent interacting with the novel object over $3 \mathrm{~min}$ (nOR) or with the object in a new location over $5 \mathrm{~min}(\mathrm{OP})$. An observer blind to experimental procedures scored all of the experiments.

\section{Social Interaction in the Open Field}

Social interaction in the open field was carried out as previously described (Kwon et al, 2006; Satoh et al, 2011). Mice were handled for 2-3 min a day for 5 days before testing. On each side of a clean square cage, a rectangular wire-holder was placed that would contain either a novel object or a stimulus mouse (C57BL/6J). Stimulus mice were habituated to the wire-holders the day before testing to prevent excessive movement. On testing day, mice to be tested (test mice) were habituated to the cage with empty wire-holders for $10 \mathrm{~min}$. During each subsequent phase, the test mouse was placed into the cage and allowed to interact freely for $5 \mathrm{~min}$. The order of presentation was as follows. Test 1: a mouse (novel mouse 1) and a novel object. Test 2: the object was immediately replaced with a novel mouse (novel mouse 2). Test 3: $24 \mathrm{~h}$ later, mouse 1 and a third novel mouse (novel mouse 3 ). Sociability was measured as the percentage of time spent interacting with the mouse $v s$ the object. Immediate and long-term social memories were measured as the percentage of time spent interacting with the novel mouse. An observer blind to experimental procedures scored all of the experiments.

\section{Open Field}

Mice freely explored an open field arena $(43.2 \times 43.2 \times$ $30.5 \mathrm{~cm}^{3}$, Med Associates, ENV-515), lit at $210 \mathrm{lux}$ and divided into 16 quadrants, for $5 \mathrm{~min}$. Locomotion was 
calculated as total quadrant entries in the open field. The number of entries in the four center quadrants, as well as the time spent (in seconds) in the four center quadrants, were taken as measures of anxiety. An observer blind to experimental procedures scored all of the experiments.

\section{Locomotor Activity}

Mice freely explored a rectangular $(20.3 \mathrm{~cm} \times 15.9 \mathrm{~cm} \times 21.3$ $\mathrm{cm}^{3}$ ) Perspex Med-Associates box (ENV-010MD) with eight infrared beams for $15 \mathrm{~min}$. The number of beam breaks was recorded throughout the session automatically.

\section{Observational Battery}

Observational battery was carried out as previously described (Crawley and Paylor, 1997; Paylor et al, 1998) at the designated time points after injection $(30 \mathrm{~min}, 24 \mathrm{~h}$ and 7 days). Body temperature was taken with a digital rectal probe (Harvard Apparatus), and physical characteristics were recorded. Each mouse was then observed in an empty cage for $1 \mathrm{~min}$, where general behavioral observations were recorded. Sensorimotor reflexes and simple motor responses were then tested in the order described in Table 1. The wire suspension test measured the latency of a mouse to fall when suspended upside down about $20 \mathrm{~cm}$ over an empty cage from a wire cagetop. In the vertical pole test, a mouse was placed facing up on a cloth-tape-covered pole $(1.5 \mathrm{~cm}$ diameter, $40 \mathrm{~cm}$ long), which was lifted to a vertical position for $1 \mathrm{~min}$. The pole test score was calculated as follows: Fell before the pole reached $45^{\circ}$ or $90^{\circ}$ angle: 0 or 1, respectively; fell in 0-10 s: 2, 11-20 s: 3, 21-30 s: 4, 31-40 s: 5, 41-50 s: 6, 51-60 s: 7; stayed on $60 \mathrm{~s}$ and climbed halfway down: 8; climbed to lower half of pole: 9; and climbed down and off in: 51-60 s: 10, 41-50 s: 11, 31-40 s: 12, 21-30 s: 13, 11-20 s: 14, 1-10 s: 15. An observer blind to experimental procedures scored all of the experiments.

Table I General Motor and Sensory Responses of Vehicle- and IGF-II-Injected Mice

\begin{tabular}{|c|c|c|c|c|c|c|}
\hline & \multicolumn{2}{|c|}{$30-\mathrm{min}$ test } & \multicolumn{2}{|c|}{ 24-h test } & \multicolumn{2}{|c|}{ 7-Day test } \\
\hline & Veh & IGF-II & Veh & IGF-II & Veh & IGF-II \\
\hline Weight & $23.0( \pm 0.40)$ & $24.83( \pm 0.48)$ & $22.8( \pm 0.53)$ & $24.0( \pm 0.52)$ & $24.0( \pm 0.65)$ & $25.67( \pm 0.7 I)$ \\
\hline Temp & $38.14( \pm 0.11)$ & $38.23( \pm 0.12)$ & $38.32( \pm 0.1)$ & $37.88( \pm 0.33)$ & $38.36( \pm 0.15)$ & $38.0( \pm 0.21)$ \\
\hline Palpebral closure (\% with) & 0 & 0 & 0 & 0 & 0 & 0 \\
\hline Exophthalmos (\% with) & 0 & 0 & 0 & 0 & 0 & 0 \\
\hline Piloerection (\% with) & 0 & 0 & 0 & 0 & 0 & 0 \\
\hline \multicolumn{7}{|l|}{ General behavioral observations (\% of subject displaying response) } \\
\hline Licking & 0 & 0 & 0 & 0 & 0 & 0 \\
\hline Rearing & 100 & 100 & 100 & 100 & 100 & 100 \\
\hline Jumping & 0 & 0 & 0 & 0 & 0 & 0 \\
\hline Defecation & 0 & 0 & 0 & 0 & 20 & 33.33 \\
\hline Urination & 0 & 16.67 & 0 & 0 & 20 & 16.67 \\
\hline Movement around cage & 100 & 100 & 100 & 100 & 100 & 100 \\
\hline \multicolumn{7}{|l|}{ Sensorimotor reflexes (\% of subjects showing normal response) } \\
\hline Righting & 100 & 100 & 100 & 100 & 100 & 100 \\
\hline Whiskers & 100 & 100 & 100 & 100 & 100 & 100 \\
\hline Pole test & $10.6( \pm 1.12)$ & $11.67( \pm 0.67)$ & $10.8( \pm 1.29)$ & $12.67( \pm 1.02)$ & $13.2( \pm 0.45)$ & $13.67( \pm 0.61)$ \\
\hline Tail suspension (\% of subjects showing normal response) & 100 & 100 & 100 & 100 & 100 & 100 \\
\hline
\end{tabular}




\section{Glucose Measurements}

Tail blood was taken at the designated time points after injection ( $30 \mathrm{~min}, 12 \mathrm{~h}, 24 \mathrm{~h}$ and 7 days). Glucose was measured using the Accu-Check Aviva Plus monitoring system (Roche, San Francisco, USA).

\section{Cannulae Implants and Drug Injection}

Cannulae implants and drug injection was performed as described previously (Fernandez et al, 2008). Mice were anesthetized with ketamine $(100 \mathrm{mg} / \mathrm{kg})$ and xylazine $(75 \mathrm{mg} / \mathrm{kg})$, and guide cannulae (C232GC; 22 gauge; Plastics One) were directed toward the hippocampus $(-1.7 \mathrm{~mm}$ posterior to bregma, $\pm 1.5 \mathrm{~mm}$ lateral to midline, $-2.3 \mathrm{~mm}$ ventral to skull surface) using a stereotaxic apparatus (Kopf Instruments). Mice recovered for at least 5 days before undergoing behavioral experiments. Drugs were delivered in $0.25 \mu \mathrm{l}$ over $45 \mathrm{~s}$ via injection through the cannula (26 gauge, extending $0.8 \mathrm{~mm}$ beyond the $1.5 \mathrm{~mm}$ guide) attached to polyethylene tubing (PE50) connected to a $10-\mu \mathrm{l}$ Hamilton syringe and controlled by a microinfusion pump (Harvard Apparatus). Cannula placement was verified at the end of the behavioral experiments, following fixation of the brains in 10\% formalin. Forty-micrometer coronal sections were cut through the hippocampus, stained with cresyl violet, and examined under a light microscope. All surgeries correctly targeted the hippocampus.

\section{Western Blotting Analysis}

Western blotting analysis was done as reported previously (Chen et al, 2011). Protein extracts from dorsal hippocampus and prefrontal cortex (including anterior cingulate cortex, prelimbic cortex, and infralimbic cortex) were obtained. Equal amounts of total protein (10 or $20 \mu \mathrm{g}$ per lane) were resolved on denaturing SDS-PAGE gels and transferred to Immobilon-FL membranes (Millipore) by electroblotting. Primary antibodies: anti-Arc (1/1000, Synaptic Systems), anti-Zif268/Egr-1 (1/1000, Cell Signaling), or anti-actin (1/10 000, Santa Cruz Biotechnology). Secondary antibodies: anti-rabbit IRDye $800 \mathrm{CW}$ and anti-mouse IRDye680 (1/10 000, Li-Cor). Membranes were scanned on the Li-Cor Odyssey imager under non-saturating conditions. Data were quantified using pixel intensities with the Odyssey software according to the protocols of the manufacturer (Li-Cor, Nebraska, USA).

\section{Statistical Analysis}

One- or two-way analysis of variance followed by Dunnett's, Newman-Keuls, or Bonferroni post hoc test or Student's $t$-test were used for statistical analyses.

\section{RESULTS}

\section{IGF-II Enhances Contextual but not Auditory Fear Conditioning}

First, we determined whether an acute, systemic treatment (s.c.) of IGF-II, like an injection given directly into the rat hippocampus (Chen et al, 2011), affects aversive memories in mice. S.c. injections were chosen because previous studies have successfully used this route of administration of IGFs in different species to affect general functions without profoundly changing glucose metabolism or body weight (Douglas et al, 1991; Guler et al, 1989; Spencer et al, 1996; Zhuang et al, 1996), even when the IGFs were injected continuously for up to 2 weeks (Conover et al, 2002; Zhuang et al, 1996). Detailed statistical analyses for all experiments shown in this manuscript can be found in Supplementary Table S1.

To establish a dose-response curve, mice were injected with $5,15,30,45,60$, or $100 \mu \mathrm{g} / \mathrm{kg}$ of IGF-II or vehicle 20 min before contextual fear conditioning (CFC) training. Fifteen, 30, and $45 \mu \mathrm{g} / \mathrm{kg}$ of IGF-II, but not the other doses, significantly enhanced CFC memory, tested $24 \mathrm{~h}$ after training (Figure 1a). A dose of $30 \mu \mathrm{g} / \mathrm{kg}$ of IGF-II showed the strongest effect on CFC memory, whereas 15 and $45 \mu \mathrm{g} / \mathrm{kg}$ produced intermediate, although significant, memory enhancement. We further investigated the effect of 15 and $30 \mu \mathrm{g} / \mathrm{kg}$ of IGF-II on CFC retention at $24 \mathrm{~h}$ and 7 days after training. A dose of $15 \mu \mathrm{g} / \mathrm{kg}$ of IGF-II significantly increased memory retention at $24 \mathrm{~h}$ and produced a very strong trend toward memory enhancement at 7 days (Figure $1 \mathrm{~b}$ ), while $30 \mu \mathrm{g} / \mathrm{kg}$ of IGF-II resulted in a more robust and significant enhancement at both $24 \mathrm{~h}$ and 7 days retention tests (Figure 1c). Thus, for all subsequent experiments we used the more effective dose of $30 \mu \mathrm{g} / \mathrm{kg}$, injected s.c., unless otherwise indicated. Furthermore, IGFII injected $20 \mathrm{~min}$ before CFC training significantly enhanced short-term memory tested at $1 \mathrm{~h}$ after training (Figure 1d), indicating that IGF-II injected before training may enhance not only memory consolidation, the process that following learning makes memory long-lasting and resilient to interferences (McGaugh, 2000), but also learning and/or short-term memory. As IGF-II injected immediately after CFC training significantly enhanced memory retention at $24 \mathrm{~h}$ (Figure 1e), we concluded that IGF-II also affects memory consolidation, in agreement with our previous findings obtained with IGF-II hippocampal injections (Chen et al, 2011). The effect of posttraining IGF-II treatment was transient, as 7 days later, the IGF-II effect was lost (Figure 1e). Compared with vehicle, the posttraining injection of IGF-II did not affect short-term memory retention tested $1 \mathrm{~h}$ after training (Figure 1f). Thus, to produce a stronger and more persistent effect on memory retention with systemic injections, IGF-II should be administered before training.

IGF-II injected 20 min before auditory fear conditioning training did not change retention either before or after the onset of the tone given at testing $24 \mathrm{~h}$ later (Figure $1 \mathrm{~g}$ ). This indicates, in agreement with our previous data with IGF-II injections directly into the rat basolateral amygdala (Chen et al, 2011), that IGF-II does not enhance amygdaladependent auditory fear conditioning and shows that freezing per se is not affected by IGF-II administration. Thus, IGF-II appears to target memories known to involve hippocampal and/or cortical regions, but not those that are more strictly amygdala dependent, suggesting specific regional or network mechanisms for IGF-II. These results indicate that IGF-II may be particularly suitable for targeting medial temporal lobe-dependent memories and cognitive and executive functions rather than amygdaladriven, Pavlovian conditioning. 

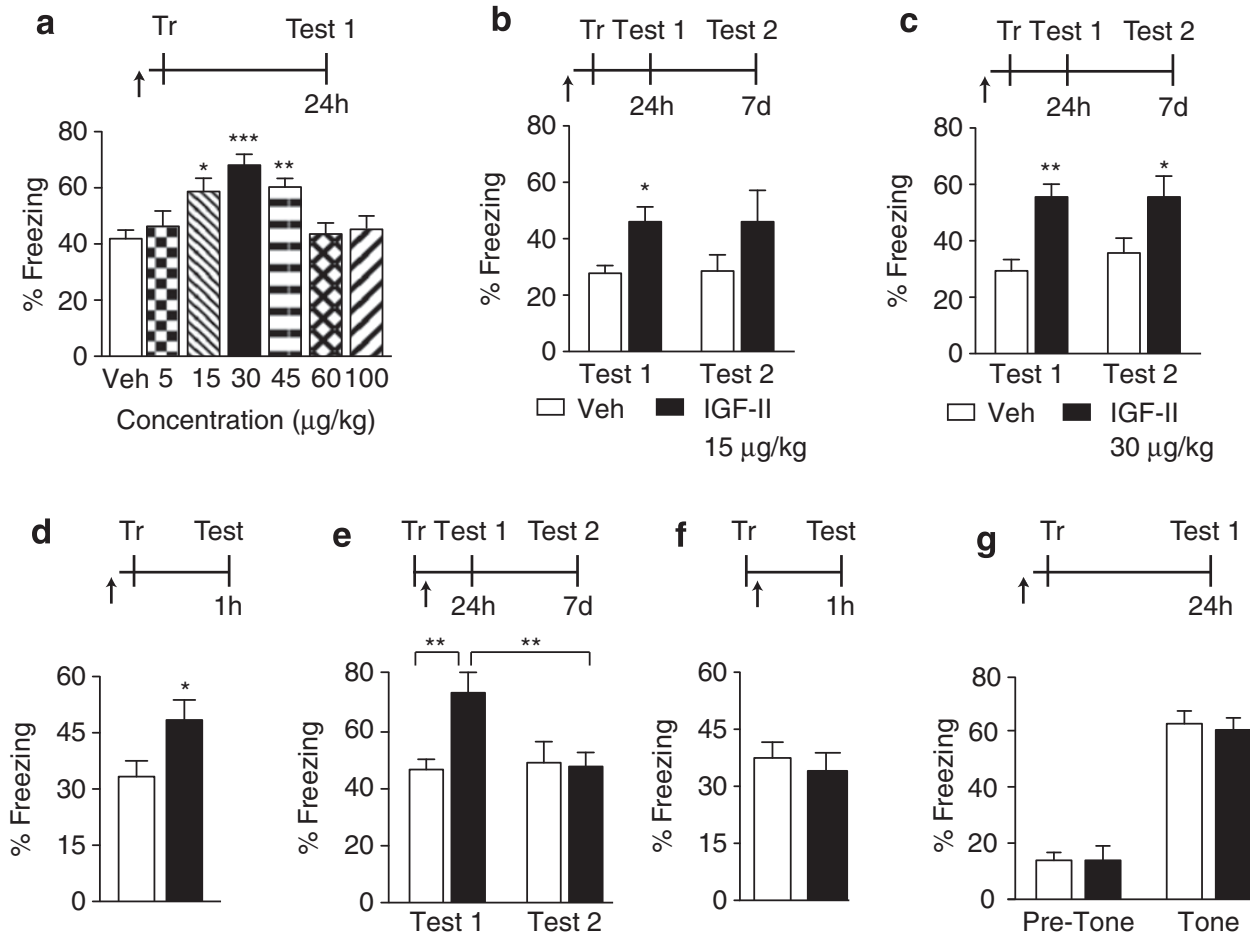

$\square$ Veh $\square$ IGF-II

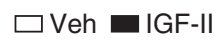

$\square$ Veh $\square$ IGF-II
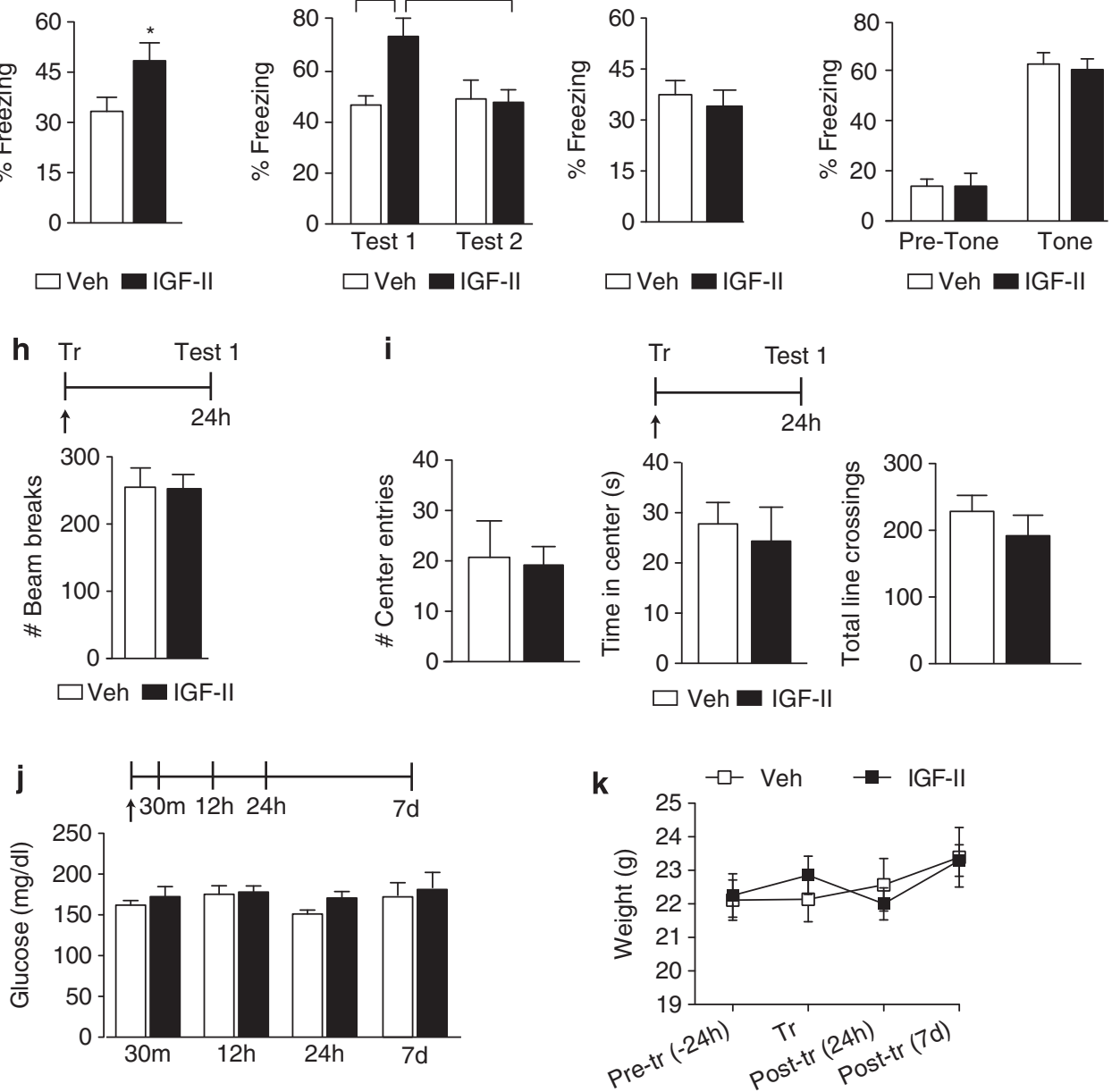

Figure I Systemic IGF-II enhances contextual fear but not auditory fear memory. Experimental schedules are shown above each panel. Tr: Training. (a-f) Data are expressed as mean ( \pm SEM) percentage (\%) freezing. Mice were injected ( $\uparrow$ ) with vehicle (Veh) or IGF-II 20 min before or immediately after training. (a) I5, 30, and $45 \mu \mathrm{g} / \mathrm{kg}$, but not other doses, of IGF-II enhances contextual fear conditioning memory $(n=6-9)$. (b) I5 $\mu \mathrm{g} / \mathrm{kg}$ IGF-II enhances memory at I day, but not at 7 days, after training $(n=7-8)$. (c) $30 \mu \mathrm{g} / \mathrm{kg}$ enhances memory at both I and 7 days after training $(n=8-1 \mathrm{I})$. (d) IGF-II enhances short-term memory, I h after training $(n=7)$. (e) IGF-II injected immediately after training enhances memory at $24 \mathrm{~h}$, but not at 7 days, after training $(n=7-8)$. (f) IGF-II injected immediately after training does not enhance short-term memory $(n=6)$. (g) IGF-II has no effect on auditory fear conditioning memory $(n=6)$. IGF-II has no effect on ( $h$ ) locomotor activity, expressed as mean ( \pm SEM) beam breaks of mice injected with vehicle (Veh) or IGF-II $24 \mathrm{~h}$ before testing $(n=5-6)$, (i) open field test, expressed as mean ( \pm SEM) center entries (Left) or time spent in the center (in s) (Middle) or number of total line crossings (Right) in the open field arena at $24 \mathrm{~h}$ after injection of vehicle or IGF-II $(n=6)$, (j) mean ( \pm SEM) of blood glucose concentration (mg/dl) $30 \mathrm{~min}, 12 \mathrm{~h}, 24 \mathrm{~h}$, and 7 days after injection of Veh or IGF-II $(n=6)$, or $(\mathrm{k})$ mean $( \pm$ SEM) weight $(\mathrm{g})$ of mice injected with either Veh or IGF-II 20 min before CFC training and tested $24 \mathrm{~h}$ before (Pre), immediately after (Tr), $24 \mathrm{~h}$ and 7 days after training (Post, $n=7$ ). $* P<0.05$, *** $P<0.0 \mathrm{l}$, **** $P<0.00 \mathrm{l}$. 


\section{Acute IGF-II Treatment does not Produce Adverse Effects}

To determine whether the IGF-II treatment produces adverse effects, we assessed the safety of the s.c. IGF-II injection by conducting a standard observational battery of tests that included physical, behavioral, and sensorimotor evaluations (Paylor et al, 1998). As shown in Table 1, no differences were found between vehicle- and IGF-II-injected mice at $30 \mathrm{~min}, 24 \mathrm{~h}$, or 7 days after injection. Furthermore, systemic IGF-II treatment did not affect general locomotor activity (Figure $1 \mathrm{~h}$ ) or the open field locomotion/anxiety test assessed at either $20 \mathrm{~min}$ or $24 \mathrm{~h}$ after injection (Supplementary Figure S1, Figure 1i). Consistent with previous reports (Conover et al, 2002; Zapf et al, 1985; Zhuang et al, 1996), IGF-II did not affect blood glucose levels tested $30 \mathrm{~min}, 12 \mathrm{~h}, 24 \mathrm{~h}$, and 7 days after IGF-II injection (Figure 1j). Additionally, IGF-II did not affect body weight measured immediately, $24 \mathrm{~h}$, and 7 days after training (Figure 1k), time points at which memory enhancement was found.

We concluded that acute systemic treatments with IGF-II are safe and that IGF-II targets memory formation and retention rather than motor or anxiety responses.

Systemically Delivered IGF-II Enhances Memory via the IGF-IIR Expressed in the Brain and Increases Brain Expression of Immediate Early Genes (IEGs)

IGF-II has been shown to cross the BBB (Duffy et al, 1988; Reinhardt and Bondy, 1994). Here we asked whether IGF-II delivered systemically affects memory via IGF-IIR expressed in the brain, as indicated previously by intra-hippocampal injections (Chen et al, 2011). Twenty minutes before CFC training, IgG or a functionally blocking IGF-IIR antibody (anti-IGF-IIR) was injected into the dorsal hippocampus of mice, followed immediately after by a vehicle or IGF-II s.c. injection (Figure 2a). Testing $24 \mathrm{~h}$ later revealed that antiIGF-IIR completely blocked memory enhancement elicited by the s.c. delivery of IGF-II. These data show that s.c.administered IGF-II exerts its effect on memory via IGF-IIR expressed in the brain.

To begin identifying the mechanisms by which a systemic injection of IGF-II affects cognitive functions, and to determine whether the s.c. route of administration influences cellular responses in the brain, we investigated whether the IGF-II injection changes the expression of IEGs known to be regulated by neural activity and/or plasticity (Guzowski, 2002). Specifically, we evaluated the effect of a $30-\mu \mathrm{g} / \mathrm{kg}$ s.c. injection of IGF-II on the expression levels of activity-regulated cytoskeleton-associated protein (Arc/ Arg3.1) and zinc finger protein Zif268 (also known as early growth response protein 1, Egr-1) in the hippocampus and prefrontal cortex of naive and trained mice. Both Arc and Zif268 are known to have an essential role in long-term plasticity and memory (Guzowski, 2002).

Quantitative western blotting analysis revealed that, compared with vehicle, an injection of IGF-II significantly augmented the expression levels of both Arc (Figure 2b) and Zif268 (Figure 2c) in the hippocampus, but not in the prefrontal cortex, of naive mice (Figure $2 \mathrm{~d}$ and e), although there was a trend toward an increase in cortical Zif268.
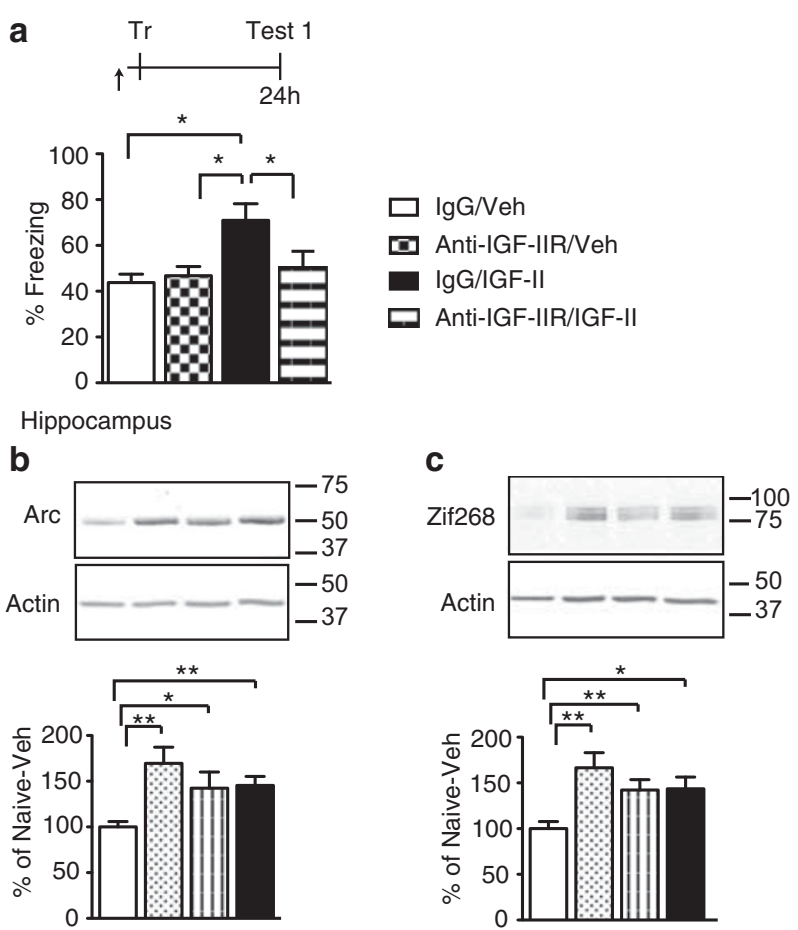

Prefrontal Cortex

\section{d}

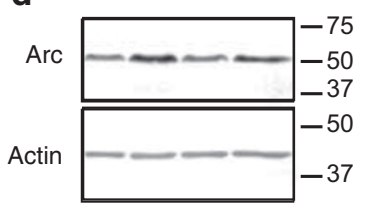

e
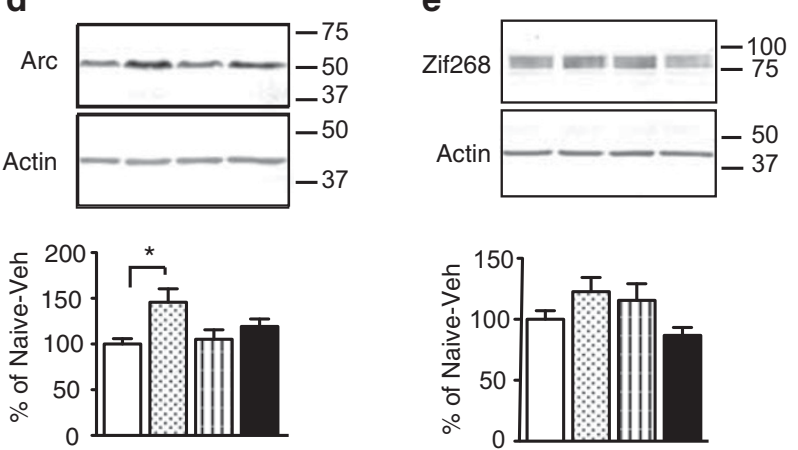

Naive-Veh Trained-Veh 미 Naive-IGF-II Trained-IGF-II

Figure 2 Systemic treatment with |GF-II enhances memory via hippocampal IGF-IIR and increases the expression of immediate early genes (IEGS) in the brain. (a) Experimental schedule is shown above the panel. Tr: Training. Data are expressed as mean ( \pm SEM) percentage $(\%)$ freezing. Mice were injected $(\uparrow)$ with IgG or anti-IGF-IIR intrahippocampally and, immediately after, with vehicle (Veh) or IGF-II s.c. Twenty min later they underwent CFC training, and were tested 24h later. (b-e) Examples and quantification of western blots of hippocampal and prefrontal cortex extracts obtained from naive and trained mice injected $(\uparrow)$ with either vehicle (Veh) or IGF-II 20 min before CFC training and euthanized I h after training. Values were normalized against actin. Data are expressed as mean percentage ( \pm SEM) of naive mice injected with Veh (Naive-Veh). In naive mice, compared with Veh, IGF-II injection (Naive-IGF-II) increases the levels of (b) Arc and (c) Zif268 in the hippocampus, but not in the prefrontal cortex (d, Arc: e, Zif268). Training significantly increases the levels of Arc in the hippocampus and prefrontal cortex and of Zif268 in the hippocampus (Trained-Veh); IGF-II treatment does not further increase the levels of either IEGs following training (Trained-IGF-II). $(n=7-\mid 0), \quad * P<0.05$, *** $P<0.01$.

Furthermore, in agreement with previous studies (Alberini, 2009; Bramham, 2007; Holloway and McIntyre, 2011; Zhang et al, 2011), training significantly elevated both Arc and 
Zif268 levels in the hippocampus (Figure $2 \mathrm{~b}$ and c) and led to a significant induction of Arc and a trend toward an increase of Zif268 in the prefrontal cortex; however, IGF-II did not further change these inductions (Figure $2 \mathrm{~d}$ and e). We concluded that systemic treatments with IGF-II modify the activity-related molecular activation of neural cells in brain regions known to critically process medial temporal lobe-dependent memory formation.

\section{IGF-II Enhances Several Types of Non-Aversive Memories and Fear Extinction}

Because many types of memory impaired in psychopathologies are non-aversive, it is important to determine the effect of IGF-II on different non-aversive tasks relevant for potential translation into clinical applications. Toward this end, we examined the effect of systemic IGF-II treatment on the nOR and OP tasks (Dix and Aggleton, 1999), which model hippocampal and cortical-dependent episodic types of memories (Antunes and Biala, 2012), known to be impaired in Alzheimer's disease (Dere et al, 2007). These tasks are based on rodents' innate preference for novelty. Compared with vehicle, injection of IGF-II 20 min before nOR training did not affect nOR memory retention at $4 \mathrm{~h}$ after training (Test 1) but significantly enhanced it at $24 \mathrm{~h}$ after training (Test 2). In fact, at Test 2, the vehicle group performed at chance level (ie, 50\%), while the IGF-IIinjected mice showed significant preference for the novel object (Figure 3a). Compared with vehicle, IGF-II significantly enhanced OP memory at both 4 and $24 \mathrm{~h}$ after training (Figure $3 \mathrm{~b}$ ). In both tasks, the IGF-II effect was not due to changes in motivation to explore, as both IGF-IIand vehicle-injected mice spent similar amounts of time exploring the objects at both training and testing (Supplementary Figure S2).

We then investigated the effect of IGF-II on a social recognition memory, a fundamental and adaptive memory in numerous species, which can be measured by examining the ability of the animal to differentiate between a social and non-social stimulus (ie, a mouse $v s$ an object) and between a novel and familiar mouse (Satoh et al, 2011). Mice were injected s.c. with either IGF-II or vehicle $20 \mathrm{~min}$ before testing their interaction with either a novel object or a novel mouse (Test 1). Five minutes later, they were tested for their immediate memory by assessing their interaction with either the previously encountered mouse or a novel mouse (Test 2). Long-term memory was subsequently tested $24 \mathrm{~h}$ after Test 2 by recording the animals' interaction between the previously encountered mouse $v s$ another novel mouse (Test 3). C57BL/6J mice are highly social and prefer to interact with a social stimulus (ie, the mouse) compared with a non-social stimulus (ie, the object), and IGF-II did not change the preference of the mice toward a mouse $v s$ an object (Test 1, Figure 3c). IGF-II also did not affect the short-term memory of the mice for a novel $v s$ the previously encountered mouse (Test 2, Figure 3c). However, at the $24 \mathrm{~h}$ test, when vehicle-injected mice did not show a preference for the mouse that they have previously encountered, IGFII-injected mice spent more time interacting with the novel mouse, indicating that their social interaction memory persisted (Test 3, Figure 3c). There was no change between the two groups in total exploration time during any of the test phases (Supplementary Figure S3).

Finally, as a previous study showed that hippocampal injections of IGF-II enhance contextual fear extinction in mice (Agis-Balboa et al, 2011) and because extinction learning is a therapeutically important behavioral approach in cognitive-behavioral therapies (Ressler and Mayberg, 2007), we tested whether extinction is enhanced by systemic administration of IGF-II. Extinction is the process by which an experience (conditioned stimulus or CS; eg, context exploration) that was previously paired with an aversive stimulus (unconditioned stimulus, or US; eg, footshock) gradually becomes weaker following repeated re-exposures to the CS in the absence of a US. Compared with vehicle, IGF-II injected $20 \mathrm{~min}$ before each daily extinction trial (CS exposure, Figure $3 \mathrm{~d}-\mathrm{f}$ ) significantly facilitated extinction, as shown by the lower freezing levels (Figure 3d), and the higher rate of extinction (Figure 3e) in the IGF-II-injected mice. A comparison of the last extinction trial confirmed that IGF-II significantly promotes extinction (Figure $3 \mathrm{f}$ ).

\section{IGF-II Potentiates Memory When Given with Reactivation}

Memory strength can be modulated not only around the initial phase of training but also by targeting retrieval or reactivation and the postretrieval process known as reconsolidation (Chen et al, 2011; Frenkel et al, 2005; Inda et al, 2011; Tronson and Taylor, 2007). We have previously shown that memory enhancement can also be achieved by injecting IGF-II into the rat hippocampus immediately after IA retrieval (Chen et al, 2011). Using the nOR task, we asked whether IGF-II injected systemically can enhance memory when given before memory reactivation. IGF-II or vehicle were injected $20 \mathrm{~min}$ before a nOR test (reactivation) given $4 \mathrm{~h}$ after training (when vehicle-injected controls still show memory). A control group of mice were injected with IGF-II in the absence of reactivation. As shown in Figure 3g, IGF-II significantly enhanced memory retention $24 \mathrm{~h}$ after training in mice that underwent reactivation but not in those that did not. These results indicate that memory reactivation reengages mechanisms that enable IGF-II to enhance memory retention (Figure $3 \mathrm{~g}$ ) and suggest that there are multiple temporal windows of intervention. No changes in total exploration time during training or testing were found (Supplementary Figure S4).

\section{IGF-II Enhances Working Memory, without Changing Behavioral Flexibility}

One concern about memory-enhancing treatments is whether the enhanced memory becomes inflexible (Floresco and Jentsch, 2011). Hence, we determined whether the IGF-II-dependent memory enhancement affected behavioral flexibility.

We first tested the rate of contextual extinction of mice that were injected with $15 \mu \mathrm{g} / \mathrm{kg}$ of IGF-II before training and had an enhanced CFC memory, compared with that of vehicle-injected controls (Figure 4a-c). Although IGF-IIinjected mice maintained significantly higher freezing throughout the extinction protocol (Figure 4a), both groups of animals extinguished at a similar rate (Figure $4 \mathrm{~b}$ and $\mathrm{c}$ ), 

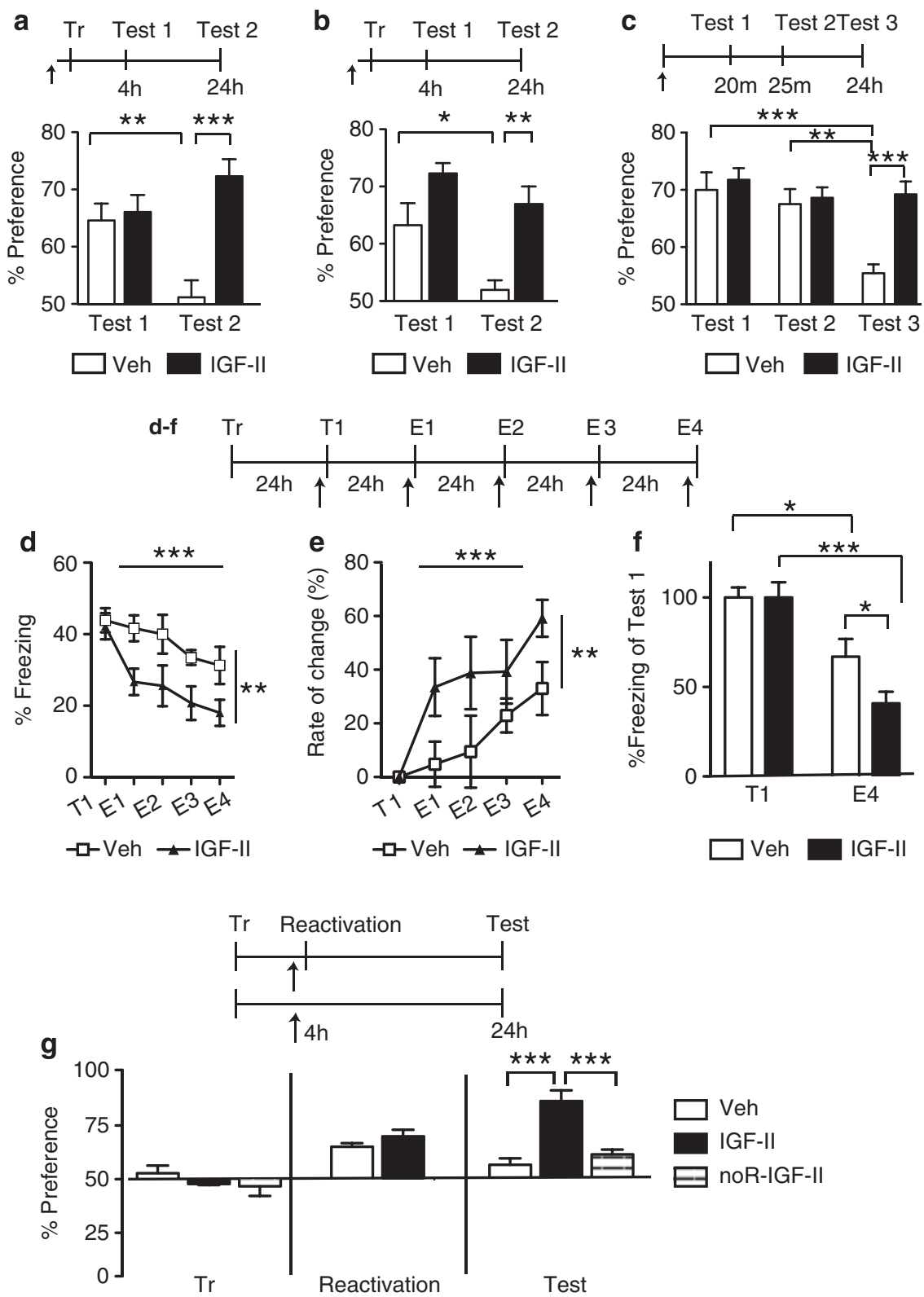

Figure 3 IGF-II increases strength and/or persistence of non-aversive memories. Experimental schedules are shown above each panel. (a) IGF-II enhances nOR memory, expressed as mean ( \pm SEM) \% preference for a novel object $(n=9-10)$ and $(b)$ enhances OP memory, expressed as mean $( \pm$ SEM) \% preference for a displaced object $(n=6-8)$ in mice injected $(\uparrow)$ with vehicle (Veh) or IGF-II 20 min before training. (c) IGF-II does not affect social recognition $20 \mathrm{~min}$ after injection (Test I) or immediate social memory tested 5 min later (Test 2). However, it enhances social learning memory tested at $24 \mathrm{~h}$ (Test 3). Data are expressed as mean $( \pm$ SEM) \% preference for a novel mouse $(n=10)$. $(d-f)$. Schematic representation of the behavioral extinction protocol. Tr: Training; TI: Test I; EI-E4: Extinction trials I-4. (d) IGF-II facilitates contextual fear extinction, expressed as mean ( \pm SEM) \% freezing $(n=9-10)$. (e) IGF-II increases the rate of extinction, expressed as mean ( \pm SEM) \% rate of change from Test I. Mice were injected with Veh or IGF- $\|$ 20 min before each extinction trial. ( $f$ ) E4 is expressed as mean ( \pm SEM) \% freezing of TI (I00\%). (g) Mean \% ( \pm SEM) preference for the novel object in mice injected with either Veh or IGF-II 20 min before a reactivation given $4 \mathrm{~h}$ after $\operatorname{Tr}$. $(n=6)$. noR, no reactivation. $* P<0.05$, $* * P<0.0$ I, **** $P<0.00$ I.

indicating that the IGF-II-enhanced CFC memory remains flexible and similarly susceptible to extinction as a normal, non-enhanced memory.

Furthermore, we used a Y-Maze paradigm to assess working memory, learning, long-term memory and reversal learning. Working memory can be evaluated by quantifying spontaneous alternation, which is considered to be an index of active retrograde working memory, because in a Y-Maze, mice generally explore the least recently visited arm and thus tend to alternate their visits between the three arms. Many brain regions, including the hippocampus, septum, basal forebrain, and prefrontal cortex, have an important role in this task (Lalonde, 2002). Compared with vehicle, an injection of IGF-II $20 \mathrm{~min}$ before spontaneous alternation testing significantly increased the alternation rate, indicating that IGF-II enhances working memory (Figure 4d). 


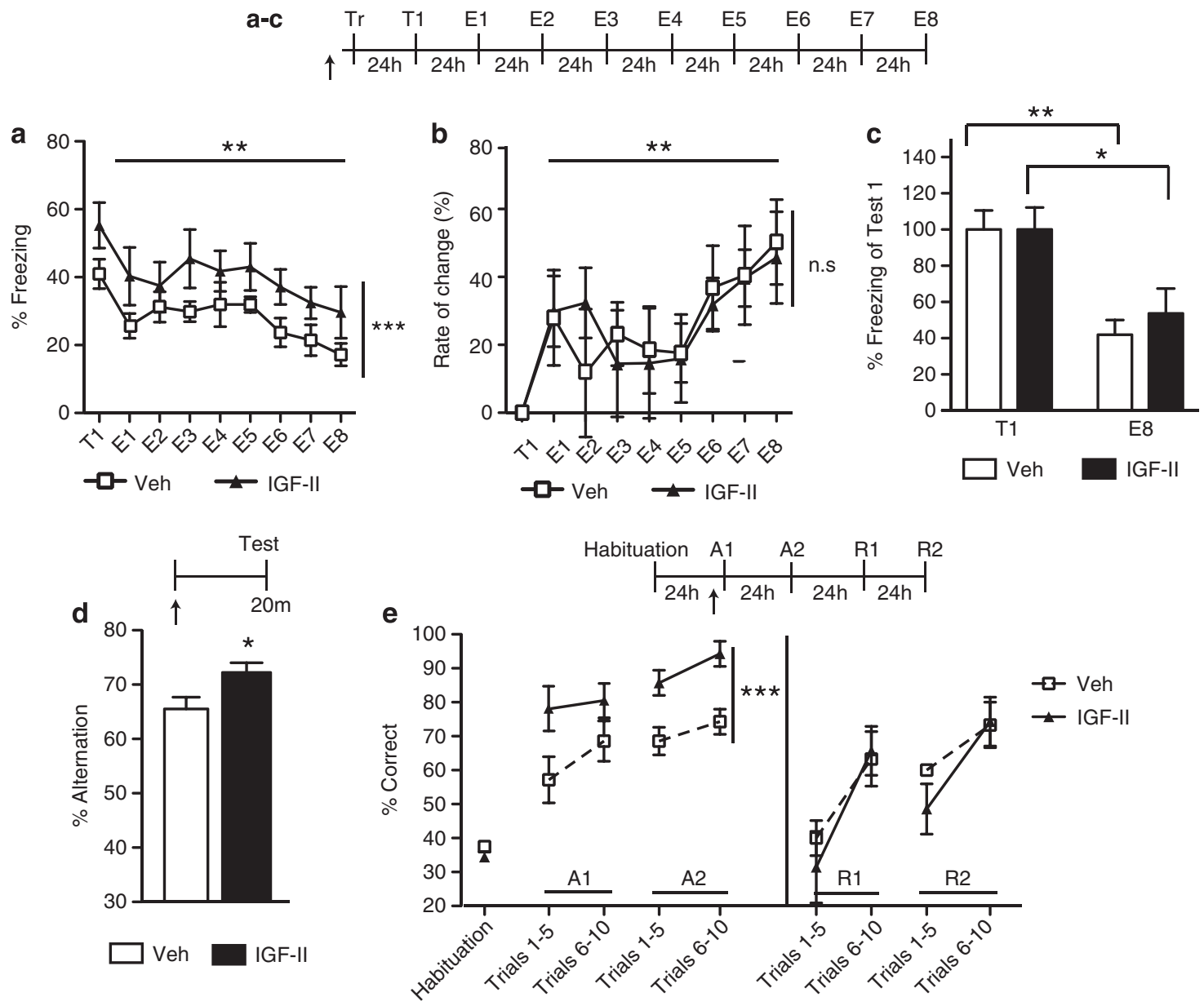

Figure 4 IGF-II enhances memory without altering its flexibility. (a-c) Schematic representation of the behavioral schedule; Tr: Training; TI:Test I; EI-E8: Extinction trials I-8. (a) CFC memory retentions in IGF-II- or vehicle (Veh)-injected ( $\uparrow$ ) mice extinguish at similar rates. Data are expressed as the mean ( \pm SEM) \% freezing $(n=8)$. (b) No difference in the rate of extinction (\%) expressed as the mean $( \pm$ SEM) rate of freezing change: [E - TI ]/TI (c) or as the $\%$ freezing of E8/TI. (d) IGF-II enhances working memory (\% alternation) in the spontaneous alternation task, expressed as the mean ( \pm SEM) \% alternation. Mice were injected with Veh or IGF-II 20 min before being placed into the Y-Maze $(n=7-8)$. (e) Spatial reference task in Y-Maze. Data are expressed as the mean $( \pm$ SEM) \% correct arm entry of mice in the Y-Maze (baited arm). AI-A2: acquisition sessions I-2; RI-R2: reversal sessions I-2; each acquisition or reversal session refers to a block of five trials $(n=7-8)(e)$. $* P<0.05$, $* * P<0.0$ l, **** $P<0.00$ I.

We then assessed reinforced spatial reference memory and reversal learning, which measures the ability to adapt to changes and inhibit the previously learned response (a form of behavioral inhibition). During habituation to the Y-Maze, mice showed no difference in preference for any one arm (Habituation, Figure 4e). Training began 1 day later and consisted of two blocks of five trials per day, given once/day for 2 days with one arm of the Y-Maze baited with a food reward. Mice were injected with IGF-II or vehicle $20 \mathrm{~min}$ before the first training session. Compared with vehicle, IGF-II significantly enhanced acquisition of the first training session (A1, Figure 4e) and a significant enhancement persisted on the second day (A2, Figure 4e). One day after the end of training, reversal learning was assessed by switching the location of the food reward to the previously unbaited arm. Mice were exposed to this reversal protocol for two blocks of five trials per day for 2 days, and both vehicle- and IGF-II-injected mice showed similar acquisition of the new location (R1 and R2, Figure 4e), confirming that IGF-II enhances working, reference, and long-term memories, without changing memory flexibility.

\section{DISCUSSION}

We showed that systemic treatments with IGF-II significantly enhance and prolong the retention of several types of short-term and long-term, aversive and non-aversive memories that are processed by hippocampal/cortical regions and are expressed by either passive or active behavior. Hence, IGF-II may be particularly suitable as an enhancer of hippocampal- or cortical-dependent memories. We also found that IGF-II significantly improves extinction and working memory without affecting behavioral flexibility. Although to our knowledge there is no report of IGFII treatments in humans, interestingly, a recent study reports an IGF-II polymorphysm associated with human cognitive functions (Alfimova et al, 2012). 
IGF-II acute systemic treatment did not have adverse effects, as demonstrated by a battery of physical, behavioral, sensimotor, and motor tests. Furthermore, IGF-II did not affect general locomotor activity or the open field locomotion/anxiety tests, body weight, or blood glucose levels at time points at which memory enhancement was detected. These data are in agreement with previous findings showing that acute actions of IGF-II on glucose metabolism in adult rat serum as well as tissues is negligible, approximately calculated to be about $2 \%$ compared with that of insulin. Furthermore, this weak effect seems to be mediated by insulin receptors (Stumpel and Hartmann, 1992). We also showed that, in agreement with previous reports indicating that IGF-II crosses the BBB (Duffy et al, 1988; Reinhardt and Bondy, 1994; Rosenfeld et al, 1987), s.c.-delivered IGF-II rapidly reaches the brain, appear to be safe, and IGF-II seems to target memory formation and retention rather than motor, anxiety, or metabolic responses. Importantly, to our knowledge, binding, distribution and actions of systemically administered IGF-II in humans remain to be determined.

In all our experiments, we found that the effect of IGF-II is rapid as revealed by the enhanced short-term memory in $\mathrm{CFC}$, OP, and working memory tasks. Furthermore, there is a limited temporal window for optimal effect, as confirmed by the fact that pretraining injections are more effective than posttraining treatments in significantly enhancing memory retention and persistence, as well as short-term behavioral responses. Because systemically administered IGF-II requires that IGF-II enters the brain, the temporal delay of posttraining injections compared to pretraining may be sufficient to partially miss the rapid IGF-IIresponsive mechanisms, hence producing a transient effect. This suggests that the time required for systemically injected IGF-II to impinge on brain functions activated by learning or a behavioral experience is key for the most effective memory enhancement. Our data show that the delivery of IGF-II 20 min before training is sufficient to capture the learning-induced mechanisms that lead to a significant and persistent memory enhancement.

These results and conclusions are also corroborated by the findings that the effect of the s.c. IGF-II treatment occurs via IGF-IIR expressed in the hippocampus and that IGF-II significantly increases the expression of Arc and Zif268 in the hippocampus $1 \mathrm{~h}$ after injection. In the prefrontal cortex, although non-significant, the increases in Zif268 may actually be relevant, as in cortical regions the network of cells recruited in memory formation is considered to be more sparse than in the hippocampus (Frankland and Bontempi, 2005). These changes in activity/ plasticity markers not only prove that the systemic treatment affects brain cellular and molecular functions but also begin to identify the mechanisms by which IGF-II affects behavior. As these IEGs are known to be activitydependent, we speculate that IGF-II may alter excitability (Silva et al, 2009), hence favoring memory strengthening and enhancement. If this is the case, our results showing no further induction of IEGs by IGF-II following training could be in line with the explanation that the activated cells may be preferentially recruited and engaged in memory processing. Because the identification of systemic treatment effects requires comprehensive approaches that survey a large number of mechanisms in the entire brain and body, future investigations are needed to determine how IEG induction and additional mechanisms lead to an increase in memory retention and persistence.

Although some mechanisms of action of IGF-II and relevant molecular correlates, including IGF-II receptors, de novo protein synthesis, GSK $3 \beta$ activation, activity-regulated cytoskeletal protein (Arc), increased synaptic GluA1, and some aspects of neurogenesis, have been identified in the hippocampus (Chen et al, 2011; Agis-Balboa et al, 2011), the means by which IGF-II may act in other brain regions, like cortices, remain to be investigated. Here we can only speculate that because IGF-II seems to be able to engage a wide range of pro-mnemonic/pro-cognitive mechanisms in the CNS, its action may either target general mechanisms of activity/plasticity or be actually pleiotropic, thus affecting distinct mechanisms in different brain regions, which may be specific for the activated trace. Furthermore, in addition to those found in the hippocampus, IGF-II mechanisms of action may include epigenetic modifications, potentiating acetylcholine release (Hawkes et al, 2006; Kar et al, 1997), attenuating GABA release (Amritraj et al, 2010), and/or fast synaptic regulation of AMPA receptors (Alberini and Chen, 2012; Chen et al, 2011).

IGF-II, like other mechanisms underlying memory consolidation and storage, including CREB activation, AMPA/ NMDA receptor trafficking, neuromodulation, and metabolic processes, seems to provide an important substrate for memory enhancement (Alberini and Chen, 2012; Stern and Alberini, 2013), and indeed these mechanisms have been targeted for developing cognitive enhancers like ampakines (Lynch, 2006) and phosphodiesterase inhibitors (Blokland et al, 2012). IGF-II offers the advantage of being a natural peptide that can be easily delivered systemically and can elicit a persistent effect after a single administration.

These results and the highly conserved structure and function of IGF-II in evolution (Duguay et al, 1995) suggest that this factor may represent an effective systemic treatment for memory and cognitive enhancement in humans.

\section{FUNDING AND DISCLOSURE}

The authors declare no conflict of interest.

\section{ACKNOWLEDGEMENTS}

This work is supported by grants R01-MH065635 and R01MH074736 awarded to CMA and F31-MH090636 awarded to SAS. We thank Virginia Gao, Mark Klinger, and the staff of the UAWC at NYU for technical support.

\section{REFERENCES}

Agis-Balboa RC, Arcos-Diaz D, Wittnam J, Govindarajan N, Blom $\mathrm{K}$, Burkhardt S et al (2011). A hippocampal insulin-growth factor 2 pathway regulates the extinction of fear memories. EMBO J 30: 4071-4083.

Alberini CM (2009). Transcription factors in long-term memory and synaptic plasticity. Physiol Rev 89: 121-145. 
Alberini CM, Chen DY (2012). Memory enhancement: consolidation, reconsolidation and insulin-like growth factor 2. Trends Neurosci 35: 274-283.

Alfimova MV, Lezheiko TV, Gritsenko IK, Golimbet VE (2012). [Association of the insulin-like growth factor II (IGF2) gene with human cognitive functions]. Genetika 48: 993-998.

Amritraj A, Rauw G, Baker GB, Kar S (2010). Leu27 insulin-like growth factor-II, an insulin-like growth factor-II analog, attenuates depolarization-evoked GABA release from adult rat hippocampal and cortical slices. Neuroscience 170: 722-730.

Antunes M, Biala G (2012). The novel object recognition memory: neurobiology, test procedure, and its modifications. Cogn Process 13: 93-110.

Blokland A, Menniti FS, Prickaerts J (2012). PDE inhibition and cognition enhancement. Expert Opin Ther Pat 22: 349-354.

Bramham CR (2007). Control of synaptic consolidation in the dentate gyrus: mechanisms, functions, and therapeutic implications. Prog Brain Res 163: 453-471.

Chen DY, Stern SA, Garcia-Osta A, Saunier-Rebori B, Pollonini G, Bambah-Mukku D et al (2011). A critical role for IGF-II in memory consolidation and enhancement. Nature 469: 491-497.

Conover CA, Johnstone EW, Turner RT, Evans GL, John Ballard FJ, Doran PM et al (2002). Subcutaneous administration of insulinlike growth factor (IGF)-II/IGF binding protein-2 complex stimulates bone formation and prevents loss of bone mineral density in a rat model of disuse osteoporosis. Growth Horm IGF Res 12: 178-183.

Crawley JN, Paylor R (1997). A proposed test battery and constellations of specific behavioral paradigms to investigate the behavioral phenotypes of transgenic and knockout mice. Horm Behav 31: 197-211.

Dere E, Huston JP, De Souza Silva MA (2007). The pharmacology, neuroanatomy and neurogenetics of one-trial object recognition in rodents. Neurosci Biobehav Rev 31: 673-704.

Dix SL, Aggleton JP (1999). Extending the spontaneous preference test of recognition: evidence of object-location and objectcontext recognition. Behav Brain Res 99: 191-200.

Douglas RG, Gluckman PD, Ball K, Breier B, Shaw JH (1991). The effects of infusion of insulinlike growth factor (IGF) I, IGF-II, and insulin on glucose and protein metabolism in fasted lambs. J Clin Invest 88: 614-622.

Duffy KR, Pardridge WM, Rosenfeld RG (1988). Human bloodbrain barrier insulin-like growth factor receptor. Metabolism 37: 136-140.

Duguay SJ, Chan SJ, Mommsen TP, Steiner DF (1995). Divergence of insulin-like growth factors I and II in the elasmobranch, Squalus acanthias. FEBS Lett 371: 69-72.

Fernandez SM, Lewis MC, Pechenino AS, Harburger LL, Orr PT, Gresack JE et al (2008). Estradiol-induced enhancement of object memory consolidation involves hippocampal extracellular signal-regulated kinase activation and membrane-bound estrogen receptors. J Neurosci 28: 8660-8667.

Floresco SB, Jentsch JD (2011). Pharmacological enhancement of memory and executive functioning in laboratory animals. Neuropsychopharmacology 36: 227-250.

Frankland PW, Bontempi B (2005). The organization of recent and remote memories. Nat Rev Neurosci 6: 119-130.

Frenkel L, Maldonado H, Delorenzi A (2005). Memory strengthening by a real-life episode during reconsolidation: an outcome of water deprivation via brain angiotensin II. Eur J Neurosci 22: 1757-1766.

Guedea AL, Schrick C, Guzman YF, Leaderbrand K, Jovasevic V, Corcoran KA et al (2011). ERK-associated changes of AP-1 proteins during fear extinction. Mol Cell Neurosci 47: 137-144.

Guler HP, Zapf J, Schmid C, Froesch ER (1989). Insulin-like growth factors I and II in healthy man. Estimations of half-lives and production rates. Acta Endocrinol (Copenh) 121: 753-758.
Guzowski JF (2002). Insights into immediate-early gene function in hippocampal memory consolidation using antisense oligonucleotide and fluorescent imaging approaches. Hippocampus 12: 86-104.

Han JH, Kushner SA, Yiu AP, Hsiang HL, Buch T, Waisman A et al (2009). Selective erasure of a fear memory. Science 323: 1492-1496.

Hawkes C, Jhamandas JH, Harris KH, Fu W, MacDonald RG, Kar S (2006). Single transmembrane domain insulin-like growth factor-II/mannose-6-phosphate receptor regulates central cholinergic function by activating a G-proteinsensitive, protein kinase C-dependent pathway. J Neurosci 26: 585-596.

Holloway CM, McIntyre CK (2011). Post-training disruption of Arc protein expression in the anterior cingulate cortex impairs longterm memory for inhibitory avoidance training. Neurobiol Learn Mem 95: 425-432.

Inda MC, Muravieva EV, Alberini CM (2011). Memory retrieval and the passage of time: from reconsolidation and strengthening to extinction. J Neurosci 31: 1635-1643.

Kar S, Seto D, Dore S, Hanisch U, Quirion R (1997). Insulin-like growth factors-I and -II differentially regulate endogenous acetylcholine release from the rat hippocampal formation. Proc Natl Acad Sci USA 94: 14054-14059.

Kwon CH, Luikart BW, Powell CM, Zhou J, Matheny SA, Zhang W et al (2006). Pten regulates neuronal arborization and social interaction in mice. Neuron 50: 377-388.

Lalonde R (2002). The neurobiological basis of spontaneous alternation. Neurosci Biobehav Rev 26: 91-104.

Lynch G (2006). Glutamate-based therapeutic approaches: ampakines. Curr Opin Pharmacol 6: 82-88.

Mandillo S, Tucci V, Holter SM, Meziane H, Banchaabouchi MA, Kallnik $M$ et al (2008). Reliability, robustness, and reproducibility in mouse behavioral phenotyping: a cross-laboratory study. Physiol Genomics 34: 243-255.

McGaugh JL (2000). Memory-a century of consolidation. Science 287: 248-251.

Paylor R, Nguyen M, Crawley JN, Patrick J, Beaudet A, OrrUrtreger A (1998). Alpha7 nicotinic receptor subunits are not necessary for hippocampal-dependent learning or sensorimotor gating: a behavioral characterization of Acra7-deficient mice. Learn Mem 5: 302-316.

Reinhardt RR, Bondy CA (1994). Insulin-like growth factors cross the blood-brain barrier. Endocrinology 135: 1753-1761.

Ressler KJ, Mayberg HS (2007). Targeting abnormal neural circuits in mood and anxiety disorders: from the laboratory to the clinic. Nat Neurosci 10: 1116-1124.

Rosenfeld RG, Pham H, Keller BT, Borchardt RT, Pardridge WM (1987). Demonstration and structural comparison of receptors for insulin-like growth factor-I and -II (IGF-I and -II) in brain and blood-brain barrier. Biochem Biophys Res Commun 149: 159-166.

Roth RA (1988). Structure of the receptor for insulin-like growth factor II: the puzzle amplified. Science 239: 1269-1271.

Russo VC, Gluckman PD, Feldman EL, Werther GA (2005). The insulin-like growth factor system and its pleiotropic functions in brain. Endocr Rev 26: 916-943.

Satoh Y, Endo S, Nakata T, Kobayashi Y, Yamada K, Ikeda T et al (2011). ERK2 contributes to the control of social behaviors in mice. J Neurosci 31: 11953-11967.

Schrick C, Fischer A, Srivastava DP, Tronson NC, Penzes P, Radulovic J (2007). N-cadherin regulates cytoskeletally associated IQGAP1/ERK signaling and memory formation. Neuron 55: 786-798.

Silva AJ, Zhou Y, Rogerson T, Shobe J, Balaji J (2009). Molecular and cellular approaches to memory allocation in neural circuits. Science 326: 391-395. 
Spencer GS, Decuypere E, Buyse J, Zeman M (1996). Effect of recombinant human insulin-like growth factor-II on weight gain and body composition of broiler chickens. Poult Sci 75: 388-392.

Stern SA, Alberini CM (2013). Mechanisms of memory enhancement. Wiley interdiscip Rev Syst Biol Med 5: 37-53.

Stumpel F, Hartmann H (1992). Acute actions of insulin-like growth factor II on glucose metabolism in adult rats. Diabetologia 35: 932-938.

Tronson NC, Taylor JR (2007). Molecular mechanisms of memory reconsolidation. Nat Rev Neurosci 8: 262-275.

Van der Borght K, Havekes R, Bos T, Eggen BJ, Van der Zee EA (2007). Exercise improves memory acquisition and retrieval in the Y-maze task: relationship with hippocampal neurogenesis. Behav Neurosci 121: 324-334.
Werther GA, Russo V, Baker N, Butler G (1998). The role of the insulin-like growth factor system in the developing brain. Horm Res 49(Suppl 1): 37-40.

Zapf J, Schoenle E, Froesch ER (1985). In vivo effects of the insulin-like growth factors (IGFs) in the hypophysectomized rat: comparison with human growth hormone and the possible role of the specific IGF carrier proteins. Ciba Found Symp 116: 169-187.

Zhang Y, Fukushima H, Kida S (2011). Induction and requirement of gene expression in the anterior cingulate cortex and medial prefrontal cortex for the consolidation of inhibitory avoidance memory. Mol Brain 4: 4.

Zhuang HX, Snyder CK, Pu SF, Ishii DN (1996). Insulin-like growth factors reverse or arrest diabetic neuropathy: effects on hyperalgesia and impaired nerve regeneration in rats. Exp Neurol 140: 198-205.

Supplementary Information accompanies the paper on the Neuropsychopharmacology website (http://www.nature.com/npp) 